\title{
Relaciones entre las aportaciones a la zona regable del río Jucar y la conductividad de la Albufera de Valencia
}

\author{
Juan M. Soria, María Sahuquillo y Rosa Miracle \\ Departamento de Microbiología y Ecología. Facultad de Ciencias Biológicas. Universidad de Valencia. \\ 46100 - Burjassot (Valencia). E-mail: juan.soria@uv.es
}

\section{RESUMEN}

Las aportaciones a la zona regable del río Júcar condicionan la conductividad de la Albufera porque recibe las aguas sobrantes de riego. Se ha observado una relación inversa entre ambas variables. La demanda de agua para otras zonas pretende satisfacerse a partir del trasvase de los sobrantes de riego basándose en una mejora de la eficiencia del regadío. Estas actuaciones deben realizarse sólo cuando se aseguren previamente las aportaciones necesarias para mantener la calidad del lago.

Palabras clave: Albufera de Valencia, conductividad, aportaciones, calidad de aguas.

\section{ABSTRACT}

The water inflows to the irrigation area of the Jucar river determine the conductivity of the Albufera because it receives the surplus irrigation water. It has been observed an inverse relationship between both variables. The water demands for other regions is sought by transferring the surplus irrigation water based on an improvement of the irrigation efficiency. These actions must be carried out only after having ensured a sufficient quantity of water inflow to maintain the quality of the lake.

Keywords: Albufera de Valencia, conductivity, water inflow, water quality

\section{INTRODUCCIÓN}

La Albufera de Valencia es una laguna costera somera (profundidad media de $1 \mathrm{~m}$ ) situada en la costa mediterránea al sur de la ciudad de Valencia. Tiene una extensión de $23.94 \mathrm{~km}^{2}$, y está rodeada de $223 \mathrm{~km}^{2}$ de arrozales (Sanjaume et al., 1992). El conjunto de la Albufera y los arrozales fueron declarados Parque Natural en 1986. Su cuenca hidrográfica tiene una extensión de $917.1 \mathrm{~km}^{2}$, desde el nivel del mar hasta una altura de unos 1000 m.s.n.m. La cuenca vierte a la Albufera por diversos barrancos. Los más importantes llegan por la zona Norte que son el de Poyo (también conocido como barranco de Torrent o barranco de Massanassa), cuya cuenca tiene $367.6 \mathrm{~km}^{2}$ (el $40 \%$ de la cuenca total), y el de Beniparrell (también conocido como barranco de Picassent). Los demás barrancos terminan en acequias de las huertas y arroza- les que acaban en la Albufera. Aunque la Albufera tiene una cuenca hidrológica propia, la realidad es que ésta sólo proporciona una pequeña parte de las aportaciones, mientras que la gran mayoría de las aguas llegan desde el río Júcar, ya sea por la Acequia Real del Júcar o por las tomas de Sueca y Cullera (Fig. 1). En este sentido, la Albufera es parte integrante del esquema hidrológico del río Júcar, pues recibe además por una red de sesenta y tres acequias el agua sobrante del riego. Estas acequias también recogen parte de los vertidos de aguas residuales de poblaciones de los alrededores. Además existen aportes de manantiales que surgen del fondo y de los alrededores de la laguna, cuyas aguas recoge también la red de acequias que cruzan los arrozales (Soria, 1997). La Acequia Real del Júcar es el canal de riego que proporciona el agua a la mayor parte de las huertas y los arrozales situados en el entorno de la Albufera; su cau- 
dal medio en los periodos de riego es de $15 \mathrm{~m}^{3} / \mathrm{s}$. La Acequia Madre de Sueca da servicio a los arrozales de la zona sur de la Albufera; su caudal medio en los periodos de riego es de $13 \mathrm{~m}^{3} / \mathrm{s}$.

Los trabajos realizados por Soria (1997), recogidos en parte en varias publicaciones (Soria et al., 2001; Soria y Vicente, 2002; Soria et al., 2002) aportan además datos en cuanto a las aportaciones de cada acequia a la Albufera, la influencia de las avenidas en las característi- cas del lago y las relaciones entre sus variables fisicoquímicas, por lo que no es necesario extenderse más sobre ello.

\section{MATERIAL Y MÉTODOS}

Desde 1987 a 2000 se dispone de los datos de conductividad medidos a intervalos de tiempo variables entre 15 y 30 días según la época del

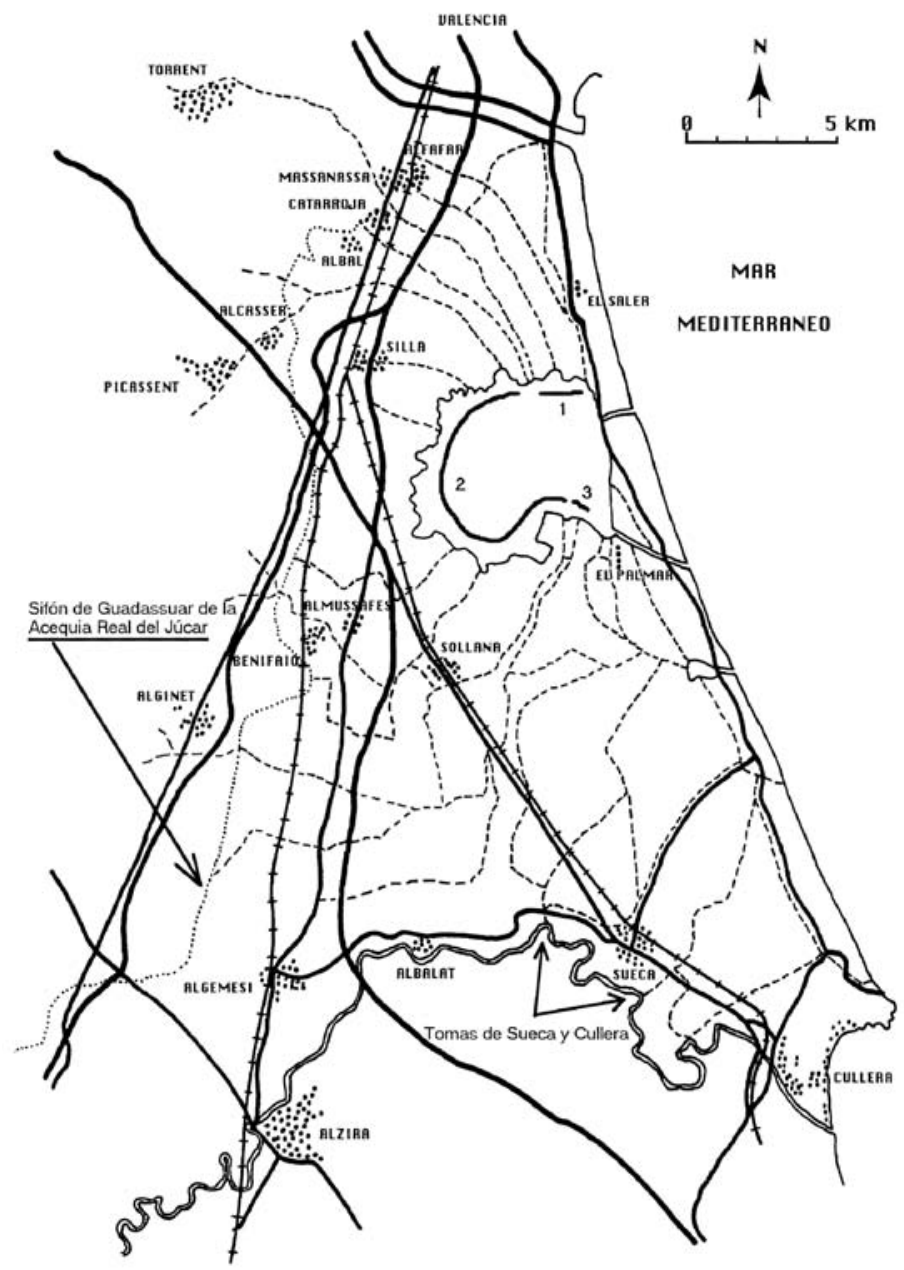

Figura 1. Esquema de la Albufera y el marjal circundante, señalando las poblaciones; las vías de comunicación en trazo contínuo; los cauces y acequias con trazos discontínuos; dentro de la Albufera se indican las diferentes zonas de influencia de aguas sobrantes de riego del río Turia con el número 1, de la Acequia Real del Júcar con el número 2 y de la acequia Madre de Sueca con el número 3. Diagram of the Albufera and the sorrounding marsh, showing the location of towns; road links are drawn with a solid line; rivers and irrigation channels are drawn with dashed lines; within the Albufera, the different influencing areas of the surplus irrigation water from the Turia river is indicated as number 1; water from the Acequia Real of Jucar river as number 2, surplus irrigation; water from Acequía Madre de Sueca as number 3. 
año para la Albufera de Valencia. Se ha tomado el valor de la conductividad superficial a $10 \mathrm{~cm}$ de profundidad por medio de un medidor de campo en siete puntos del lago distribuidos regularmente tratando de abarcar las diferentes zonas del mismo. Los datos originales proceden de varias fuentes (Soria, 1997; y datos no publicados de la Consellería de Medio Ambiente de la Generalitat Valenciana), con un total de 849 mediciones. Posteriormente se ha calculado la media mensual a partir de las diferentes medidas puntuales realizadas en un mes natural, y por último la media anual por años naturales. Con la misma agregación anual, se han obtenido las aportaciones en origen a la Acequia Real (medidas en el aforo de Antella, origen de la acequia) y al denominado cano de Sueca (origen de la acequia Madre de Sueca), a partir de la suma de los caudales suministrados diariamente registrados por el Organismo de Cuenca (la Confederación Hidrográfica del Júcar). Los datos se han procesado en hojas de cálculo por medio del programa Excel de Microsoft.

El objetivo del trabajo es estudiar qué relación puede existir entre la conductividad que se mide en el lago y las aportaciones en origen de su tributario principal de la zona agrícola, cuyo

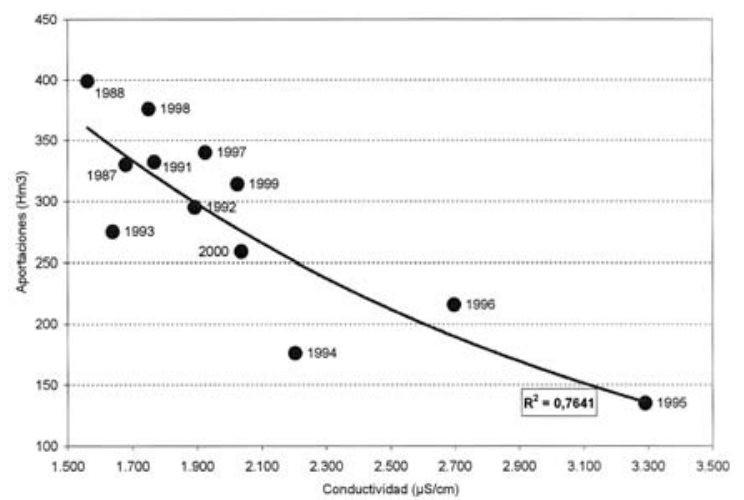

Figura 2. Representación gráfica de los valores de conductividad frente a las aportaciones en origen a la Acequia Real del Júcar para cada uno de los años, así como línea de ajuste de la regresión entre ambos valores. Graphic representation of the conductivity values regarding the water inflow from the Acequia Real of Jucar river for each year; also shown the regression line between both values. valor de conductividad permanece constante a lo largo del tiempo y la influencia sobre la salinidad del lago que puede tener una gestión inadecuada de las aportaciones.

\section{RESULTADOS}

La figura 2 representa los valores de las aportaciones anuales a la Acequia Real del Júcar frente a los valores de la conductividad media anual de la Albufera. El ajuste de la regresión entre los valores representados proporciona un coeficiente de correlación de 0.764 , significativo estadísticamente para un valor de probabilidad superior al $99 \%$. Esto nos permite afirmar que existe una correlación negativa entre las aportaciones en origen a la acequia Real y la conductividad media anual que presenta el lago de la Albufera. El mismo proceso de datos con las aportaciones en Sueca no muestra correlación significativa.

De acuerdo con los datos de Soria (1997), la mayor parte de las escorrentías a la Albufera procedentes de la Acequia Real lo hacen por las acequias de Albal, Beniparrell, Silla, L'Alqueresia, Campets y Overa, que suponen el $53 \%$ de las aportaciones al lago, y un $17 \%$ para otros cincuenta canales y acequias de menor importancia. Las aguas procedentes de sobrantes de la zona del Turia suponen el $18 \%$ de las aportaciones y la acequia Dreta el $12 \%$. La presencia de esta distribución zonal irregular de los afluentes produce una marcada heterogeneidad espacial en la Albufera que hace que circulen más aprisa las aguas por unas zonas que por otras, de la forma representada esquemáticamente en la figura 3. Así, las aguas procedentes de las acequias Overa y Dreta, que suponen el $57 \%$ de las entradas a la Albufera, salen al lago en la zona indicada como Sur y salen hacia el mar por la Sequiota y la Gola del Perellonet, por lo que su influencia en las aguas del resto del lago son inapreciables. El $18 \%$ de las aportaciones llegan a la Albufera por la zona Norte, que son las procedentes del río Turia y las depuradoras. Esta agua circula hacia la salida al mar por la Gola del Pujol. El resto de las aportaciones, el $25 \%$ 


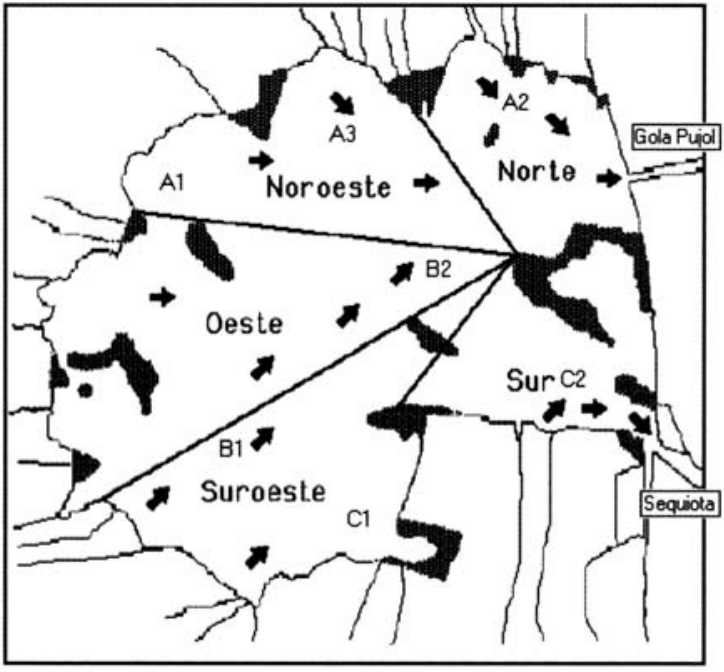

Figura 3. División de la Albufera en cinco zonas, señalando los fitoestromas internos y las direcciones de los flujos del agua en el lago y los puntos de muestreo. Division of the Albufera in five areas, showing the internal islands of vegetation, the direction of the water flow within the lake, and the sampling points.

son las que aportan las aguas al $80 \%$ de la superficie de la Albufera, en la zona Oeste. La renovación de las aguas tiene lugar, calculada a partir de los datos de Soria (1977), cada veinte días en la zona Norte, ciento veinte días en la zona Oeste y diez días en la zona Sur.
Por último, debemos señalar que la distribución de estas aportaciones y sobrantes de riego no es regular en el tiempo. Los resultados de los trabajos anteriormente citados de Soria y Vicente (2002) y Soria et al., (2001) muestran que tras los periodos de lluvias se alcanzan los momentos de mayor aportación y los valores más bajos de conductividad, mientras que los sobrantes de agua son inexistentes durante el verano en la época del cultivo del arroz, cuando incluso se producen flujos inversos desde la Albufera hacia los arrozales, elevando el agua de los canales con bombas hasta los campos. En esos momentos, desde mayo hasta agosto, las aportaciones son prácticamente inexistentes, y se produce un aumento de la conductividad hasta el doble de la existente en invierno, como se refleja en el estudio de Soria (1997), así como en los datos obtenidos en estos últimos tres años, representados en la figura 4. Por el contrario, durante la época de lluvias torrenciales y crecidas en el barranco del Poyo (el tributario mayor natural de la Albufera), las aportaciones son muy elevadas y los descensos de conductividad más acusados (Soria et al., 2001), como ha sucedido en febrero de 1998 y octubre de 2000. En este trabajo citado se muestra el hidrograma de crecida en la rambla de Poyo y se ve cómo las

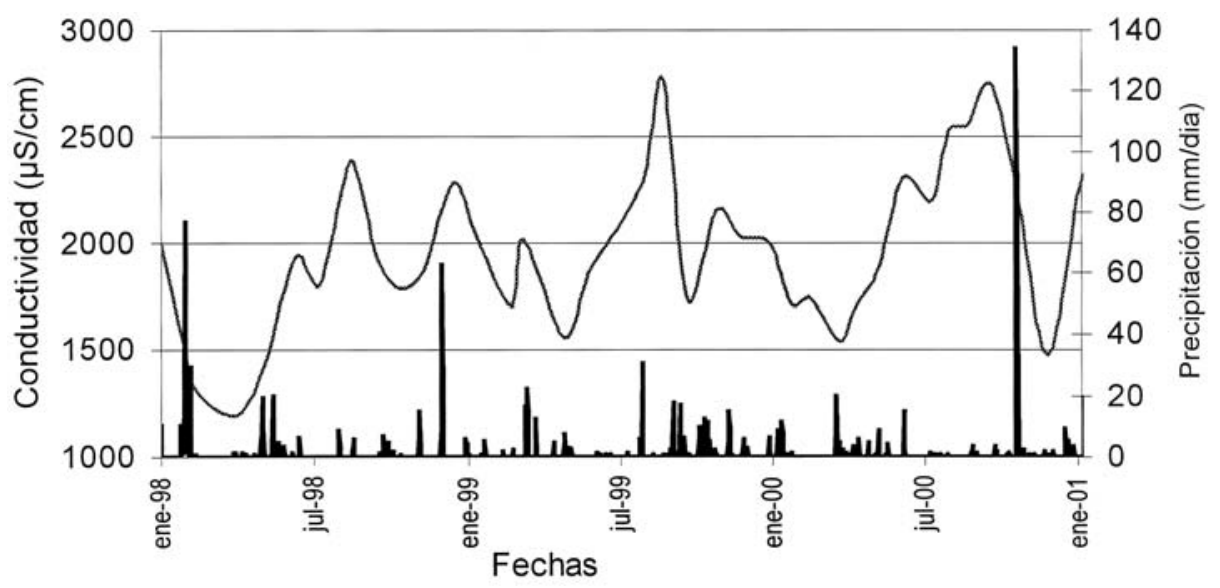

Figura 4. Ciclo anual de la conductividad y precipitaciones en la Albufera en los años 1998, 1999 y 2000. Annual cycle of conductivity and rainfall within the Albufera in 1998, 1999 and 2000. 
aportaciones duran poco más que los días de la crecida y la semana siguiente a lo sumo. El incremento de volumen producido es aliviado rápidamente al mar favorecido por las estaciones de bombeo construidas en las Golas por el Ayuntamiento de Valencia en los años 90 con la finalidad que el lago y el marjal próximo recupere un nivel de agua que considera razonable la Junta de Desagüe de la Albufera, organismo que gestiona los desagües. Esto supone que las aguas de mejor calidad procedentes de las escorrentías naturales no llegan a distribuirse por todo el lago.

\section{DISCUSIÓN}

El Plan Hidrológico del Júcar (CHJ, 1997) establece unos aportes mínimos de aguas procedentes del río Júcar de $100 \mathrm{hm}^{3}$ anuales para la Albufera. Sin embargo, se pudo evaluar a lo largo de 1988 la aportación anual al lago en un volumen de $280 \mathrm{hm}^{3}$ (Soria, 1997). Todavía se desconoce la distribución que tendrá ese volumen de agua a lo largo del ciclo anual, así como los cauces que llevarán el agua hasta el lago. Las posibles consecuencias que un descenso de las aportaciones puede tener sería un aumento de la conductividad general del lago a corto plazo. Tampoco existe un modelo de calidad del lago en cuanto a conductividad, pero se estima en base al valor medio de la conductividad de las aportaciones que no deberían superarse los valores máximos de $2000 \mu \mathrm{S} / \mathrm{cm}$ en los momentos más desfavorables. Cualquier disminución de los caudales en origen influirá en la salinización del lago, que se produce tanto por la disminución del aporte del agua dulce al mismo procedente de los sobrantes de riego, como por entrada por infiltración del agua marina al cesar la presión de salida del agua dulce, tal y como se describe en el estudio de Hidrogeología de la Albufera realizado para el Ayuntamiento de Valencia (Soria, 1993), y como sucedió el año 1995, cuando se alcanzaron valores puntuales de hasta $8.000 \mu \mathrm{S} / \mathrm{cm}$ y promedio mensual de
$5.000 \mu \mathrm{S} / \mathrm{cm}$ por causa de desaparecer completamente las aportaciones de todo tipo. Es precisamente la gran superficie del lago de la zona Oeste la que más pérdida de aportaciones sufrirá cuanto mayor sea el descenso de los sobrantes como consecuencia de los cambios de los sistemas de riego y mejora en las eficiencias de la distribución del agua de riego. La consecución de un grado de calidad aceptable en la Albufera pasa por el aporte de aguas en cantidad razonable, especialmente por la zona Oeste, dependiente de la acequia Real del Júcar. Si el volumen de aportación actual (datos del autor de 2003 no publicados) está en el orden de los $50 \mathrm{hm}^{3}$ por esta zona, y la previsión de la Administración Hidráulica es disminuir estas aportaciones por la mejora del regadío, la calidad del agua del lago se vería afectada negativamente al aumentar la conductividad. Eliminar esos $50 \mathrm{hm}^{3}$ de aportaciones para ser trasvasado a otra cuenca podría producir en los próximos años una salinización del lago al disminuir la presión del agua dulce.

\section{CONCLUSIONES}

La conductividad de la Albufera aparece relacionada inversamente por una parte con las aportaciones de aguas en origen a la Acequia Real del Júcar y por otra con las aportaciones naturales en su cuenca durante los periodos de lluvias importantes. Para garantizar la calidad de las aguas de la Albufera en un nivel de conductividad razonable, que estimamos no debería superar los $2000 \mu \mathrm{S} / \mathrm{cm}$ por tratarse de una laguna dulce, se deben mantener las aportaciones de aguas del río Júcar previstas por el Plan Hidrológico y conseguir que su distribución se realice en los periodos que más lo necesite el lago. Asimismo, esta distribución debe hacerse de forma regular por todos los tributarios naturales al mismo, especialmente los que desembocan en la zona Oeste para conseguir una renovación más homogénea del lago. El trasvase de aguas de riego no debe producirse mientras no se asegure un nivel de calidad en el lago. 


\section{BIBLIOGRAFÍA}

CONFEDERACIÓN HIDROGRÁFICA DEL JÚCAR. 1997. Plan Hidrológico del Júcar. 112 pp.

SANJAUME, E., F. SEGURA, M. J. LÓPEZ y J. PARDO. 1992: Tasas de sedimentación en L'Albufera de València. Cuad. de Geogr., 51: 63-81.

SORIA, J. M. 1993. Caracterización fisicoquímica de las surgencias del Parque Natural de la Albufera (Valencia). Actas VI Congreso Español de Limnología: 93-97.

SORIA, J. M. 1997. Estudio limnológico de los ecosistemas acuáticos del Parque Natural de la
Albufera de Valencia, Tesis Doctoral. Universidad de Valencia. 292 pp.

SORIA, J. M., E. VICENTE \& M. R. MIRACLE. 2001. The influence of flash floods on the limnology of the Albufera of Valencia lagoon (Spain). Verh. Internat. Verein. Limnol., 27: 2232-2235.

SORIA, J. M. y E. VICENTE. 2002. Estudio de las aportaciones al Parque Natural de la Albufera de Valencia. Limnetica, 21: 105-116

SORIA, J. M., MIRACLE, M. R. \& E. VICENTE. 2002. Relations between physic-chemical and biological variables in aquatic ecosystems of the Albufera Natural park (Valencia, Spain). Verh. Internat. Verein. Limnol., 28: 564-568. 